Documentation et bibliothèques

\title{
Une société à documenter : les acquis et les défis
}

Society's Need for Information: Accomplishments and

Challenges

\section{Una sociedad a documentar: las realizaciones y los desafíos}

\section{Richard Paré}

Volume 38, numéro 3, juillet-septembre 1992

URI : https://id.erudit.org/iderudit/1028621ar

DOI : https://doi.org/10.7202/1028621ar

Aller au sommaire du numéro

\section{Éditeur(s)}

Association pour l'avancement des sciences et des techniques de la documentation (ASTED)

\section{ISSN}

0315-2340 (imprimé)

2291-8949 (numérique)

Découvrir la revue

Citer cet article

Paré, R. (1992). Une société à documenter : les acquis et les défis.

Documentation et bibliothèques, 38(3), 135-138.

https://doi.org/10.7202/1028621ar

\section{Résumé de l'article}

Le besoin d'information dans notre société actuelle est inévitable et l'information est maintenant devenue une denrée quotidienne. Pour combler ce besoin, les citoyens peuvent compter sur un ensemble de services d'archives et de bibliothèques développés au cours des ans. Ils peuvent aussi exploiter des nouvelles technologies de l'information et de la documentation engendrées par l'usage courant des ordinateurs. La présente réflexion brosse, à grands coups de pinceaux, les grandes lignes des acquis dans la fresque documentaire québécoise et canadienne et pose plus particulièrement les défis à relever, compte tenu des effets que les nouvelles technologies de l'information auront de plus en plus sur la documentation.
Tous droits réservés ( $)$ Association pour l'avancement des sciences et des techniques de la documentation (ASTED), 1992
Ce document est protégé par la loi sur le droit d'auteur. L’utilisation des services d'Érudit (y compris la reproduction) est assujettie à sa politique d'utilisation que vous pouvez consulter en ligne.

https://apropos.erudit.org/fr/usagers/politique-dutilisation/ 


\title{
Une société à documenter: les acquis et les défis
}

\author{
Richard Paré* \\ Président \\ ASTED
}

Le besoin d'information dans notre société actuelle est inévitable et linformation est maintenant devenue une denrée quotidienne. Pour combler ce besoin, les citoyens peuvent compter sur un ensemble de services d'archives et de bibliothèques développés au cours des ans. I/s peuvent aussi exploiter des nouvelles technologies de l'information et de la documentation engendrées par l'usage courant des ordinateurs. La présente réflexion brosse, à grands coups de pinceaux, les grandes lignes des acquis dans la fresque documentaire québécoise et canadienne et pose plus particulièrement les défis à relever, compte tenu des effets que les nouvelles technologies de linformation auront de plus en plus sur la documentation.

\section{Society's Need for Information: Accomplishments and Challenges}

Society's need for information is very real and information is now considered to be a precious resource used daily. In order to satisfy their need, citizens can turn to the services of the archives and libraries that have developped over the years. They can also avail themselves of the new sources of information accessible by computer. The following article summarises the accomplishments of libraries in Québec and throughout Canada and identifies tomorrow's challenges that will be coloured by the new information technologies.
Una sociedad a documentar: las realizaciones y los desafios

La necesidad de información en nuestra sociedad actual es inevitable y la información es ahora un producto cotidiano. Para satisfacer esta necesidad, los ciudadanos pueden contar con un conjunto de servicios de archivos y de bibliotecas desarrollados con el correr de los años. Pueden también explotar nuevas tecnologías de la información y de la documentación engendradas por el uso corriente de las computadoras. La presente reflexión bosqueja, a grandes pinceladas, las grandes líneas de las realizaciones en el cuadro documental quebequense y canadiense y plantea más particularmente los desafíos a aceptar,tomando en cuenta los efectos que las nuevas tecnologias de la información tendrán, cada vez más, sobre la documentación.
Lorsque le Comité d'organisation du $19^{e}$ congrès de l'ASTED, lequel se tiendra à Montréal du 28 au 31 octobre 1992, a proposé au Conseil d'administration le thème exposé en titre de cet article, plusieurs membres se sont étonnés de son libellé. Certains ont plutôt suggéré une société «à informer» puisque nous vivons aujourd'hui dans une société d'information. D'autres membres soutenaient que la société actuelle est «plongée» dans un bain d'information dont la majeure partie est fournie à partir de multiples supports; d'où l'importance de l'accès à la documentation. Autrement dit, l'information est obtenue à partir de données stockées sur des supports dont l'ensemble constitue la documentation. Et, forcenous est de constater que les bibliothèques et les centres de documentation deviennent aujourd'hui de plus en plus des entreprises d'information documentaire.

De plus, un thème qui tenait compte du passé en incluant les acquis, se justifiait facilement puisque l'année 1992 marque l'anniversaire de plusieurs événements qui ont jalonné l'histoire de la société nordaméricaine, dont les plus significatifs sont le $350^{\mathrm{e}}$ anniversaire des villes de Montréal et de Sorel et le $125^{\circ}$ anniversaire de la Confédération canadienne. Citons en plus, le $500^{\circ}$ anniversaire de l'arrivée de Christophe Colomb; le $495^{\circ}$ anniversaire de l'arrivée de Jean Cabot sur la côte est du Canada; le $240^{\circ}$ anniversaire de la publication du premier journal canadien, le Halifax Gazette; le $225^{\circ}$ anniversaire de la fondation de la première bibliothèque publique, celle du Collège de Montréal, et enfin le $200^{\circ}$ anniversaire du parlementarisme canadien.

Puisque la proposition d'un tel thème provoquait déjà des discussions animées au Conseil d'administration et alimentait des controverses, on a conclu qu'il nourrirait sûrement la réflexion et qu'il favoriserait les échanges durant le congrès. Le thème «Une société à documenter: les acquis et les défis» fut donc retenu.

\section{Les acquis}

Lorsque l'on songe aux acquis en matière de documentation en milieu francophone au Québec et au Canada, il nous vient immédiatement à l'esprit les divers réseaux de bibliothèques et de dépôts d'archives qui constituent l'infrastructure sur laquelle s'appuient les individus, les entreprises privées et les organismes publics pour s'alimenter en sources d'information primaire et, par la suite, pour produire une information renouvelée ef différente.

\footnotetext{
* Richard Paré est bibliothécaire parlementaire associé à la Bibliothèque du Parlement du Canada.
} 
De cette infrastructure documentaire il faut identifier immédiatement les cinq piliers qui en forment la base: la Bibliothèque nationale du Canada, la Bibliothèque nationale du Québec, I'Institut canadien de l'information scientifique et technique, les Archives nationales du Canada et, enfin, les Archives nationales du Québec. Ces cinq piliers constituent à notre avis la pierre angulaire de l'ensemble du réseau documentaire au Québec et au Canada.

Par leur service du dépôt légal, les deux bibliothèques nationales reçoivent la grande majorité des ouvrages publiés au Québec et au Canada et en diffusent les notices aux citoyens et à toutes les bibliothèques par le biais de leur bibliographie courante. Ces notices permettent d'alimenter les bases de données internationales et de promouvoir ainsi l'information et la documentation québécoise et canadienne dans les pays étrangers.

Un autre acquis de base sont les archives qui constituent la mémoire collective d'un peuple ou d'une nation.

Les Archives nationales du Canada furent établies en 1872 et les Archives de la province de Québec en 1920. Ces dernières sont devenues les Archives nationales du Québec en 1969. Qu'elles soient privées ou gouvernementales, civiles ou judiciaires, audiovisuelles, photographiques, sonores, iconographiques, cartographiques, architecturales ou héraldiques, les archives servent à documenter les recherches qui conduiront à la production de nouvelles informations et de nouveaux documents.

En plus des archives, les industries du patrimoine, particulièrement les musées, produisent une documentation faisant ressortir les valeurs historiques de notre société. Notons, comme acquis, le jumelage des fonctions de muséologie, de bibliothéconomie et d'art d'interprétation dans des bibliothèques publiques du Québec particulièrement. Citons aussi la bibliothèque du Musée des Beaux-Arts du Canada et celle du Musée du Québec.

À l'actif, il faut également souligner la constitution de grands réseaux de bibliothèques et de centres de documentation lesquels ont permis la concertation, la collaboration et des économies de res- sources. L'avènement de l'informatique dans les diverses opérations d'une bibliothèque a facilité la formation de ces réseaux. Le partage des données du catalogage et de la classification et la mise en chantier de catalogues collectifs furent à l'origine des réseaux de bibliothèques tant au Canada que dans toute l'Amérique du Nord.

Par exemple, la Conférence des recteurs et principaux des universités du Québec (CREPUQ) a confié à son souscomité des bibliothèques, depuis maintenant vingt-cinq ans, la coordination du développement en réseau des bibliothèques universitaires québécoises.

L'implantation du système RENARD dans les bibliothèques de collèges du Québec a permis de relier entre elles la majorité des bibliothèques de Cégep.

De même, grâce à l'implantation du réseau des bibliothèques centrales de prêts $(B C P)$, les services d'une bibliothèque publique sont devenus accessibles aux petites municipalités du Québec.

L'accès aux services des grandes banques de données internationales, qu'elles soient des banques d'information numérique, bibliographique ou textuelle, constitue l'une des plus grandes réalisations de notre ère. II permet d'identifier, de localiser et parfois même d'obtenir très rapidement un document dont on a besoin.

À ce titre, citons le Service de recherche DOBIS de la Bibliothèque nationale du Canada dont la base de données renferme maintenant plus de sept millions de notices. Les abonnés de Dobis se retrouvent au Québec $(21,9 \%)$, en Ontario $(42,2 \%)$ et dans les autres provinces et territoires $(35,9 \%)^{1}$.

On peut avancer qu'en 1992, si quelque chercheur a besoin d'une publication ou d'un document déjà enregistré dans une banque de données reconnue, il peut se le procurer dans un délai raisonnablement court et à un prix relativement abordable.

Àce bilan, s'ajouteaussi ce réseau des bibliothèques scolaires et collégiales du Québec qu'on dénomme souvent médiathèques puisque, pour un grand nombre d'entre elles, on y intègre la documentation imprimée à la documentation audiovisuelle. La formation de ce réseau et la mise sur pied d'un Service des bibliothèques d'enseignement responsable du développement et de l'implantation des politiques et d'une Centrale des bibliothèques chargée de mettre sur pied des services en commun remontent aux années 60. Les Services documentaires multimedia (SDM Inc.) ont maintenant remplacé la Centrale des bibliothèques et continuent de fournir les services requis par le réseau des bibliothèques d'enseignement.

II y aurait encore beaucoup à dire sur le plan des acquis documentaires tant au Canada qu'au Québec, mais il faut penser aussi aux défis que l'avenir nous réserve.

\section{Les défis}

Si l'on peut dire que la télévision et, par conséquent, l'image ont eu un impact significatif dans toutes les couches de la société nord-américaine durant la deuxième moitié du $20^{\mathrm{e}}$ siècle, on peut suggérer, sans risquer de se tromper, que c'est l'informatique et plus particulièrement la micro-informatique qui auront le plus d'influence sur cette même société dans la première moitié du $21^{\circ}$ siècle.

Nous sommes à l'orée d'une deuxième vague informatique que l'OCDE, dans un récent ouvrage intitulé Villes et technologies nouvelles, qualifiait «d'informatique cognitive distribuée». Au plan des technologies de l'information, notre société s'apprête à subir une nouvelle mutation, c'est-à-dire à vivre dans le quotidien les effets des systèmes informatiques permettant l'intégration des données, du texte, de la voix et de l'image. Les applications de ces nouvelles technologies auront des effets sur les services éducatifs et culturels ainsi qu'un impact significatif sur le plan économique et social.

Les penseurs et les futuristes actuels s'entendent pour dire que la société d'information et les technologies sous-jacentes créeront de nouveaux besoins éducatifs et

1. Nouvelles de la Bibliothèque nationale, vol. 24, no 5 (mai 1992), 8. 
culturels en même temps qu'elles offriront de nouveaux moyens et de nouvelles solutions aux problèmes existants.

Que nous réserve donc l'avenir et quels sont les défis que nous devrons affronter pour nous assurer que la société sera bien documentée?

Bien avisé est celui qui saurait prédire exactement ce qui se passera dans les secteurs des bibliothèques et de la documentation. On peut, cependant, noter certains faits et souligner certaines tendances qui pourraient affecter la société de demain. Les résultats de récentes enquêtes nous ont permis de constater qu'une fraction importante de la population adulte canadienne était analphabète. Une enquête menée par Statistique Canada en 1989 a révélé que « $38 \%$ des Canadiens âgés de 16 à 69 ans ne peuvent pas répondre à leurs besoins de lecture», et «même dans le groupe plus jeune (16-24 ans) des personnes nées au Canada, le taux d'analphabétisme fonctionnel était extrêmement élevé, même si en théorie elles devaient toutes avoir fait au moins neuf années d'études obligatoires. Plus de $28 \%$ des personnes de ce groupe n'atteignaient pas le niveau de lecture courant et plus de $44 \%$ étaient incapables de compter» ${ }^{2}$.

II faudra donc favoriser des mécanismes correctifs, comme la promotion de la lecture, qui permettront de corriger cette situation. Le réseau des bibliothèques ou médiathèques scolaires et les bibliothèques publiques devraient, à notre avis, être mis davantage à contribution pour participer à la solution de ce problème d'analphabétisme. Une note encourageante cependant: une récente enquête sur les habitudes de lecture des Canadiens, menée en 1991, a enregistré une augmentation de $88 \%$ à $94 \%$ du nombre d'individus qui lisent pour leur plaisir depuis 1978. L'augmentation a été marquée surtout en Colombie-Britannique, en Nouvelle-Écosse et en Ontario ${ }^{3}$. Ce résultat constitue un signe encourageant pour le monde de l'édition et des bibliothèques.

Un autre fait qu'il nous faut bien reconnaître est celui que nous vivons maintenant dans une société d'information, c'est-à-dire dans une société où l'information est une ressource fondamentale; sa qualité et son accessibilité sont plus importantes que son support. II ne faut donc pas confondre l'information avec les moyens par lesquels elle est stockée ou véhiculée, telles les bases de données et les télécommunications. II faut donc se rendre compte de l'évolution et de l'importance du rôle de l'information en tant que ressource stratégique et de son impact sur l'infrastructure de la société d'aujourd'hui.

Lors d'un récent séminaire sur la gestion stratégique des technologies de l'information dans le secteur public, nous avons pu constater que le vocabulaire avait changé et, dans un sens, était plus dynamique que précédemment. Ainsi, plutôt que de parler de "changement», on parlait de «révolution», révolution politique, révolution technologique, révolution organisationnelle. Un certain nombre de questions au sujet des systèmes d'information ont retenu notre attention: la variété des sources de données, la dissémination dans le public, le partage des données par diverses agences, l'augmentation de l'intérêt concernant la confidentialité et la vie privée, les produits d'information faits sur mesure et les structures flexibles des bases de données.

II faut se rappeler que la technologie est seulement un outil. Elle constitue un excellent moyen de production, de dissémination et de diffusion de l'information, mais elle n'est pas une fin en soi et, à notre avis, ne devrait jamais le devenir. Le plus important consiste en l'organisation et la structure des divers domaines de la connaissance et la façon dont celle-ci peut être accessible aux utilisateurs.

Les défis de notre société à documenter tournent autour des trois axes que sont la multiplication des documents, les nouvelles technologies et les réseaux documentaires.

Nous en sommes maintenant arrivés à une société d'information, plus particulièrement en Amérique du Nord et dans les autres pays industrialisés. Aussi produit-on plus de documents que jamais, que ce soit par l'édition ou par la multiplication des supports d'information. Tous ces documents, il faut en assurer la collecte, le classement, l'indexation, l'accès à l'inventaire bibliographique. Et encore n'a-t-on pas parlé de la documentation ou littérature "grise», c'est-à-dire non pu- bliée. Bref, le défi sera de taille: il faudra faire face à la prolifération de toute cette documentation et la rendre accessible sur demande.

Parmi les nouvelles technologies auxquelles il faudra s'ajuster rapidement, nous retrouvons, dans l'immédiat, le disque optique compact (DOC ou CD-ROM), et le télécopieur et, dans un avenir rapproché, le vidéodisque et l'édition électronique.

Le disque optique compact est appelé à faire rapidement une percée dans les réseaux de bibliothèques puisqu'il permet l'accès à des masses d'informations documentaires et de documents à un coût raisonnablement bas si l'on tient compte des économies réalisées auprès des serveurs des banques de données. II est surtout rentable pour des masses d'informations et de documents qui ne nécessitent pas une mise à jour continue mais plutôt périodique.

Selon Gérard Mercure ${ }^{4}$, les producteurs de disques optiques sont présentement confrontés à trois problèmes: les coûts de traitement et de présentation de l'information, le développement des logiciels d'interrogation et le versement de droits d'auteur élevés. Cependant, nous croyons que ces problèmes vont s'atténuer rapidement et que les bibliothèques et centres de documentation devront s'adapter progressivement à l'utilisation de ce nouveau médium.

Nous ne nous attarderons point à présenter la technologie de la télécopie. Soulignons seulement que le télécopieur permet maintenant de transmettre des documents dans des délais très courts à des coûts relativement bas. Le défi en bibliothèque est de savoir l'utiliser de façon appropriée en tenant compte des coûts et des économies de temps.

2. Conseil économique du Canada, Les chemins de la compétence: éducation et formation professionnelle au Canada, Ottawa, 1992, p. 10.

3. La lecture et les Canadiens en 1991, Ottawa, Les Associés de recherche Ekos, 1991, p.15.

4. Gérard Mercure, «Le CD-ROM un portrait de famille», Argus, vol. 20, no 3 (hiver 1991), 7. 
À moyen terme, il faut s'attendre à une percée du vidéodisque qui permettra de stocker et de donner accès à de grandes quantités de documents audiovisuels.

Quant àl'édition électronique, elle aura sans aucun doute un impact sur le développement des collections des grandes bibliothèques. On la définit généralement comme la production d'un ouvrage pour la consommation générale mais dont le résultat ou le produit est lu par l'intermédiaire d'un ordinateur et visionné sur écran cathodique. L'édition électronique est aussi synonyme d'édition sans papier, d'édition numérique ou d'édition sur disque optique.

Déjà, quelques grandes revues scientifiques offrent en plus de l'édition imprimée, des abonnements à l'édition électronique. On peut citer en exemple les titres suivants: Scientific American, Chemical Week, Aviation Week et Space Technology.

Lorsque les coûts des abonnements à l'édition électronique décroîtront grâce, pour une bonne part, à une utilisation croissante et à la concurrence, il y a lieu de croire qu'elles deviendront populaires dans les grandes bibliothèques universitaires et spécialisées.

Les coûts croissants d'acquisition et de stockage des livres et documents forceront les bibliothèques et centres de documentation à former des partenariats dans le domaine du développement des collections. Comme nous l'avons vu plus haut dans "les acquis», les réseaux de bibliothèques permettent l'accès rapide à l'information bibliographique et la localisation des documents. Ces réseaux et les services qui s'y rattachent se sont, jusqu'à présent, développés à travers le Québec et le Canada surtout par genre de bibliothèques et de centres de documentation.

Il est à prévoir que de nouveaux réseaux documentaires se trouveront, non par genre de bibliothèques, mais plutôt par région. Cela permettra une meilleure planification du développement des collections et la mise en place de moyens d'entreposage en commun.

Cette tendance a déjà été perçue en 1990 par Rolande Blair lorsqu' elle souli- gnait «une activité croissante au niveau local et régional qui devrait résulter en une demande accrue pour une infrastructure décentralisée d'appuiauxactivités de mise en commun des ressources ${ }^{5}$. Le défi sera d'amener plus de bibliothèques et de centres de documentation de divers types à créer entre eux dans une même région des alliances en vue d'une meilleure mise en commun des ressources disponibles. Déjà des consortiums de services communs de bibliothèques ont vu le jour au Manitoba et en Colombie-Britannique.

Le phénomène de concertation est déjà amorcé dans le secteur du développement des collections, plus particulièrement dans le groupe de bibliothèques universitaires et de recherche. Le modèle américain "Conspectus» ${ }^{6}$ dont on se sert pourrait éventuellement s'appliquer à l'ensemble des bibliothèques canadiennes.
En conclusion, souhaitons que tous les spécialistes de l'information et de la documentation d'aujourd'hui sauront conjointement conjuguer leurs efforts pour relever les défis de la société d'information actuelle qui doit continuer à se documenter, tout en mettant en valeur les acquis du passé.

5. Rolande Blair, "Le prêt entre bibliothèques au Canada: stratégies et plan), Documentation et bibliothèques, vol. 36, no 4 (octobre-décembre 1990), 134.

6. “Conspectus» est un modèle américain développé dans les années 1970 qui deviendra l'instrument de base du North American Collections Inventory Project auquel l'Association of Research Libraries recommanda à ses membres de participer au milieu des années 1980 .

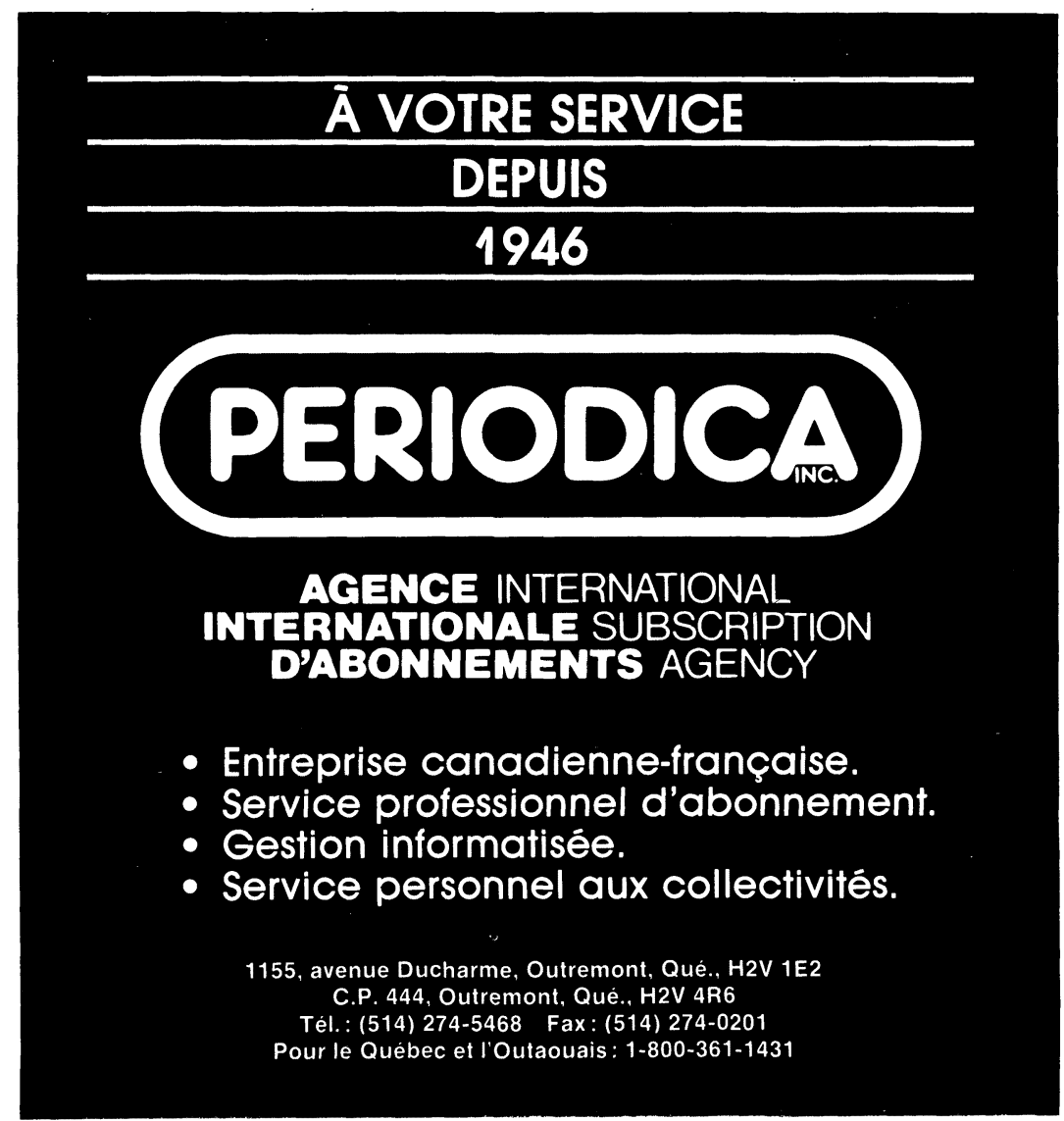

